\title{
Overexpression of the long noncoding RNA CCAT1 promotes metastasis via epithelial-to-mesenchymal transition in lung adenocarcinoma
}

\author{
HEPING LIN, WEI CHENG, HANHAN YAN and XIAODIAO ZHANG \\ Department of Respiratory Medicine, The Third Affiliated Hospital of Wenzhou \\ Medical University, Rui'an, Wenzhou, Zhejiang 325200, P.R. China \\ Received December 10, 2016; Accepted February 12, 2018
}

DOI: $10.3892 / \mathrm{ol} .2018 .8813$

\begin{abstract}
The long noncoding RNA (lncRNA) colon cancer-associated transcript 1 (CCAT1) has been identified as an oncogene in multiple types of human malignancy, and the aberrant expression of CCAT1 has been associated with the tumorigenesis and progression of cancer. However, the underlying mechanism of how CCAT1 affects malignant behaviors in lung adenocarcinoma cells remains unknown. In the current study, the expression of CCAT1 was identified to be increased in lung adenocarcinoma tissues $(n=96)$ by reverse transcription-quantitative polymerase chain reaction (RT-qPCR), and its expression level was associated with epidermal growth factor receptor (EGFR) expression $(\mathrm{P}=0.011)$, lymphatic metastasis $(\mathrm{P}=0.003)$ and tumor node metastasis (TNM) stage $(\mathrm{P}=0.003)$. In vitro, by using Transwell assays, the overexpression of CCAT1 was demonstrated to promote the migration and invasion of H358 lung adenocarcinoma cells; while downregulation of CCAT1 expression inhibited H1650 cell migration and invasion. Furthermore, western blot analysis indicated that aberrant CCAT1 expression may induce epithelial-to-mesenchymal transition (EMT) by regulating the expression levels of EMT markers (E-cadherin, N-cadherin and vimentin). In conclusion, these results indicate that CCAT1 is able to promote the metastasis of lung adenocarcinoma cells by inducing EMT.
\end{abstract}

Correspondence to: $\mathrm{Dr}$ Xiaodiao Zhang, Department of Respiratory Medicine, The Third Affiliated Hospital of Wenzhou Medical University, 108 Wansong Road, Rui'an, Wenzhou, Zhejiang 325200, P.R. China

E-mail: cindy_cici@126.com

Key words: long noncoding RNA, lung adenocarcinoma, colon cancer-associated transcript 1 , metastasis, epithelial-mesenchymal transition

\section{Introduction}

Lung cancer is one of the most common causes of cancer-associated mortality and has amongst the highest morbidity rates of all types of malignancy in China (1). Non-small cell lung cancer (NSCLC) accounts for 80-85\% of all lung cancer cases, with lung adenocarcinoma being the most common type (2). Irrespective of the progress made in diagnosis and therapy, the prognosis of patients with lung adenocarcinoma remains poor (2). A primary feature of lung adenocarcinoma is the migration and invasion of neoplasms, which are responsible for the high mortality rate (3). Therefore, understanding the mechanisms underlying metastasis in lung adenocarcinoma may reveal novel therapeutic targets.

Colon cancer-associated transcript 1 (CCAT1), also known as CARLo-5 (onco-lncRNA-40), is a long noncoding RNA (lncRNA) whose gene is located at chromosome 8q24.21, and was originally identified as an oncogene in colon cancer (4). Aberrant expression of CCAT1 in cancer cells significantly affects cell-cycle regulation and tumor development, and CCAT1 expression has been shown to predict the prognosis of patients with various tumor types (5-8). However, little is known about the expression levels of CCAT1 in lung adenocarcinoma or whether this lncRNA is involved in the progression of lung adenocarcinoma.

In the present study, the expression level and the function of CCAT1 in lung adenocarcinoma were investigated. The results demonstrated that CCAT1 was upregulated in lung adenocarcinoma tissues, and promoted the migration and invasion of lung adenocarcinoma cells by inducing epithelial-to-mesenchymal transition (EMT).

\section{Materials and methods}

Patients and specimens. Lung adenocarcinoma tissues $(\mathrm{n}=96$, aged 39-80 years; mean, 60.0 years) and pair-matched adjacent normal tissues were obtained from patients diagnosed with lung adenocarcinoma of tumor-node-metastasis (TNM) stage I-III. All patients were diagnosed between August 2010 and August 2011 at The Third Affiliated Hospital of Wenzhou Medical University (Wenzhou, China). The exclusion criteria included previous diagnosis with respiratory system diseases, 
metastasis and patients receiving radiotherapy or chemotherapy prior to their enrollment in the present study. In order to examine tumor cell concentration, frozen sections from all resected fresh tissues underwent histological analyses, and only samples with a tumor cell concentration $>60 \%$ were included in the present study. Samples were immediately frozen in liquid nitrogen and stored at $-80^{\circ} \mathrm{C}$ until use. Informed consent was obtained from each patient, and the study was approved by the Ethics Committee of The Third Affiliated Hospital of Wenzhou Medical University. Patient data are summarized in Table I.

Cell culture. Human lung cancer cell lines (H358, H23, H1975, H1650, H1299 and A549) and a normal human bronchial epithelial cell line (16HBE) were obtained from the Cell Bank of Type Culture Collection of the Chinese Academy of Sciences (Shanghai, China). Cells were cultured in Invitrogen RPMI-1640 medium with $10 \%$ fetal bovine serum (FBS) and $100 \mathrm{U} / \mathrm{ml}$ penicillin (all from Gibco; Thermo Fisher Scientific, Inc., Waltham, $\mathrm{MA}, \mathrm{USA}$ ) at $37^{\circ} \mathrm{C}$ in humidified air with $5 \% \mathrm{CO}_{2}$.

Reagents and transfection. cDNA of human CCAT1 gene was synthesized by PrimeScript RT Master Mix kit (Takara Bio, Inc., Otsu, Japan). The cDNA encoding CCAT1 was amplified by polymerase chain reaction (PCR) and subcloned into the pcDNA3.1 vector (Invitrogen, Thermo Fisher Scientific, Inc.). The empty vector was used as a negative control. All primary antibodies E-cadherin (cat no. SAB4503751), N-cadherin (cat no. SAB5500023), vimentin (cat no. SAB4503083) and goat anti-rabbit IgG secondary antibody (A0545) were purchased from Sigma-Aldrich (Merck KGaA, Darmstadt, Germany). The anti-human siRNA specifically targeting CCAT1 (synthesized by Thermo Fisher Scientific, Inc.) was used: 5'-CGGCAGGCATTAGAGATGAACAGCA-3'; and the scramble siRNA was used: 5'-CCUACGCCACCAAUU UCGU-3'. Cells were transfected using a Lipofectamine 3000 kit (Thermo Fisher Scientific, Inc.) according to the manufacturer's protocol. Cells were transfected for $48 \mathrm{~h}$ prior to their use in CCAT1 expression analyses.

Reverse transcription-quantitative PCR (RT-qPCR). Total RNA from the collected tissues was extracted using TRIzol reagent (Thermo Fisher Scientific, Inc.) according to the manufacturer's protocol, and cDNAs from all samples were synthesized by PrimeScript RT Master Mix kit (Kusatsu, Japan) and incubated for $30 \mathrm{~min}$ at $37^{\circ} \mathrm{C}, 5 \mathrm{sec}$ at $85^{\circ} \mathrm{C}$, and then maintained at $4^{\circ} \mathrm{C}$. The expression of CCAT1 was quantified using a Quantifast SYBR-Green RT-qPCR kit (Qiagen, $\mathrm{GmbH}$, Hilden, Germany). The primer sequences used were as follows: CCAT1 forward, 5'-CATTGGGAAAGGTGCCGA GA-3'; CCAT1 reverse, 5'-ACGCTTAGCCATACAGAGCC-3'; $\beta$-actin forward, 5'-AGCGAGCATCCCCCAAAGTT-3'; and $\beta$-actin reverse, 5'-GGGCACGAAGGCTCATCATT-3'. PCR was then performed at $94^{\circ} \mathrm{C}$ for $2 \mathrm{~min}$, followed by 40 cycles of $95^{\circ} \mathrm{C}$ for $10 \mathrm{sec}$ and $60^{\circ} \mathrm{C}$ for $30 \mathrm{sec}$. Each experiment was repeated independently three times. Gene expression levels were analyzed using the $2^{-\Delta \Delta \mathrm{Cq}}$ method (9).

Transwell assay. For the migration and invasion assays, $48 \mathrm{~h}$ after transfection, $3 \times 10^{4}$ cells in serum-free RPMI-1640 medium were placed into the upper chamber of each Transwell
Table I. The associations between CCAT1 expression and clinicopathological parameters.

\begin{tabular}{|c|c|c|c|c|}
\hline \multirow[b]{2}{*}{ Characteristics } & \multirow{2}{*}{$\begin{array}{l}\text { No. of } \\
\text { patients }\end{array}$} & \multicolumn{2}{|c|}{$\begin{array}{c}\text { CCAT1 } \\
\text { expression, } \mathrm{n}\end{array}$} & \multirow[b]{2}{*}{ P-value } \\
\hline & & Low & High & \\
\hline Age (years) & & & & 0.231 \\
\hline$\leq 60$ & 80 & 40 & 40 & \\
\hline$>60$ & 32 & 20 & 12 & \\
\hline Sex & & & & 0.860 \\
\hline Male & 57 & 31 & 26 & \\
\hline Female & 55 & 29 & 26 & \\
\hline EGFR & & & & 0.011 \\
\hline Negative & 74 & 46 & 28 & \\
\hline Positive & 38 & 14 & 24 & \\
\hline pT & & & & 0.539 \\
\hline 1 & 47 & 28 & 19 & \\
\hline 2 & 58 & 29 & 29 & \\
\hline 3 & 6 & 3 & 3 & \\
\hline 4 & 1 & 0 & 1 & \\
\hline $\mathrm{pN}$ & & & & 0.003 \\
\hline 0 & 63 & 43 & 20 & \\
\hline 1 & 11 & 4 & 7 & \\
\hline 2 & 35 & 11 & 24 & \\
\hline 3 & 3 & 2 & 1 & \\
\hline pTNM stage & & & & 0.003 \\
\hline I & 53 & 36 & 17 & \\
\hline II & 21 & 12 & 9 & \\
\hline III & 38 & 12 & 26 & \\
\hline
\end{tabular}

Data were analyzed using the $\chi^{2}$ test. CCAT 1 , colon cancer-associated transcript 1; EGFR, epidermal growth factor receptor; $\mathrm{pT}$, pathological tumor stage; $\mathrm{pN}$, pathological node stage; pTNM stage, pathological Tumor-Node-Metastasis stage.

insert (8.0 $\mu \mathrm{m}$; Millipore, Merck KGaA, Darmstadt, Germany). The chambers were pre-coated with Matrigel (Sigma-Aldrich; Merck KGaA, Darmstadt, Germany) for the invasion assays. The chambers were then incubated for $24 \mathrm{~h}$ at $37^{\circ} \mathrm{C}$ in a $5 \%$ $\mathrm{CO}_{2}$ incubator, with RPMI-1640 containing $10 \%$ FBS in the bottom chambers. Following incubation, cells on the upper surface were scraped off with a cotton swab and washed away with PBS (Invitrogen; Thermo Fisher Scientific, Inc.) and the invaded cells on the lower surface were fixed in $100 \%$ precooled methanol for $10 \mathrm{~min}$ and stained with $0.5 \%$ crystal violet for $2 \mathrm{~h}$. Finally, the number of invaded cells relative to the control group was calculated by counting three fields per membrane under an inverted biological microscope (magnification, $\mathrm{x} 200$ ). Experiments were independently repeated in triplicate.

Western blot analysis. Cells were lysed in RIPA buffer (Sigma-Aldrich; Merck KGaA) supplemented with a protease inhibitor (Roche, Basel, Switzerland) and a phosphatase inhibitor (Roche). Protein concentration was measured using a 

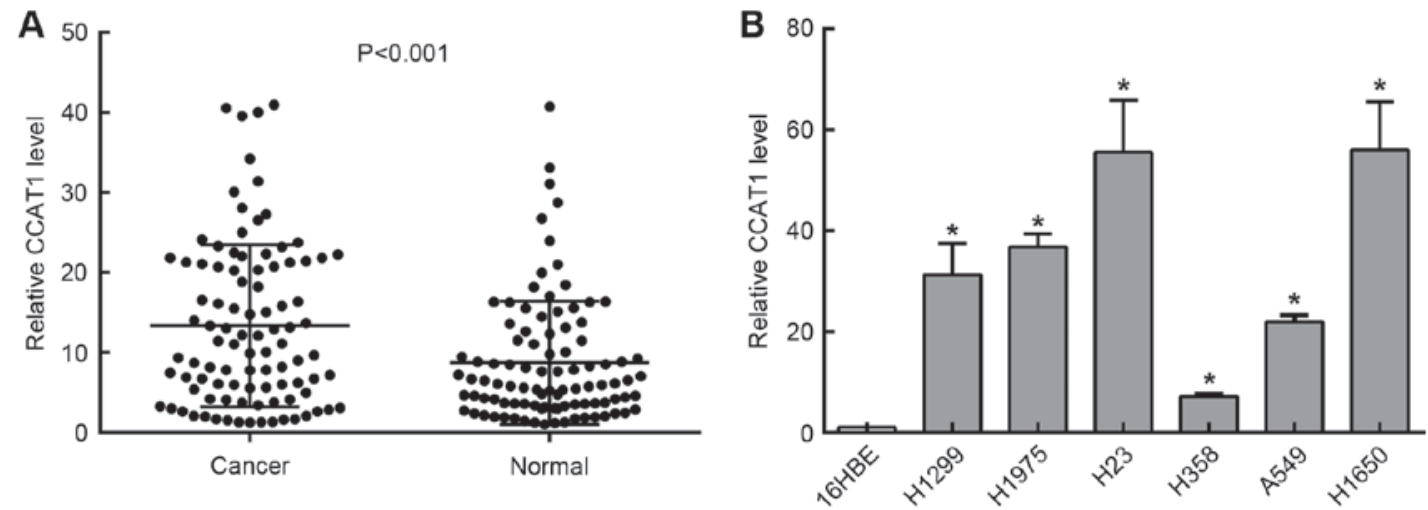

Figure 1. CCAT1 expression in lung adenocarcinoma tissues and cell lines. (A) Expression levels of CCAT1 in human lung adenocarcinoma tissues and corresponding adjacent tissues determined by RT-qPCR ( $\mathrm{n}=96$, analyzed by Student's t-test). The mean expression level of CCAT1 in lung adenocarcinoma tissues was significantly higher than that in corresponding non-malignant lung tissues. $\beta$-actin was used as an internal control. (B) Expression level of CCAT1 in lung adenocarcinoma cell lines determined by RT-qPCR. The mean expression level of CCAT1 in lung adenocarcinoma cells was significantly higher than that in the human bronchial epithelial cell line 16HBE. $\beta$-actin was used as an internal control. "P<0.01 vs. 16HBE (analyzed by one-way ANOVA). CCAT1, colon cancer-associated transcript 1; RT-qPCR, reverse transcription-quantitative polymerase chain reaction.

BCA protein Assay kit (Thermo Fisher Scientific, Inc.). Total protein lysates $(25 \mu \mathrm{g})$ were fractionated by $10 \%$ sodium dodecyl sulfate-polyacrylamide gel electrophoresis (SDS-PAGE), and were electrophoretically transferred to polyvinylidene difluoride membranes (Roche). Enhanced chemiluminescence (Tiangen Biotech Co., Ltd., Beijing, China) was used to visualize the antibody binding. Equal protein loading was controlled by using a mouse anti- $\beta$-actin monoclonal antibody. The membranes were blocked with $10 \%$ non-fat milk in Tris-buffered saline Tween (TBST) for $2 \mathrm{~h}$ at room temperature, washed and then probed with the primary antibodies (dilution 1:100) overnight at $4{ }^{\circ} \mathrm{C}$, followed by incubation with the goat anti-rabbit IgG secondary antibody conjugated to horseradish peroxidase (dilution 1:2,000, TBST dilution) for $2 \mathrm{~h}$ at room temperature. The membranes were then washed three times for $15 \mathrm{~min}$ with PBST. Proteins were detected using a Molecular Imager ChemiDoc XRS System (Bio-Rad Laboratories, Inc., Hercules, CA, USA). The integrated densities of the bands were quantified by ImageJ version software (v1.7.0, National Institutes of Health, Bethesda, MD, USA).

Statistical analysis. Statistical analysis was performed and presented using GraphPad Prism version 6.0 (GraphPad Software Inc., La Jolla, CA, USA). The data obtained from in vitro assays were analyzed by paired Student's t-test, $\chi^{2}$ test, Wilcoxon rank-sum (Mann-Whitney) test or one-way ANOVA followed by Tukey's multiple comparison tests, as appropriate. All tests performed were two-sided. $\mathrm{P}<0.05$ was considered to indicate a statistically significant difference.

IncRNA CCAT1 is upregulated in human lung adenocarcinoma tissues and cell lines. The expression levels of CCAT1 were analyzed in cancer tissues $(\mathrm{n}=96)$ and adjacent normal tissues from patients with lung adenocarcinoma by RT-qPCR. The results indicated that CCAT1 expression, relative to $\beta$-actin, was significantly higher in lung adenocarcinoma tissues than adjacent normal tissues ( $\mathrm{P}<0.001$; Fig. 1A). To further investigate the associations between clinicopathological features and CCAT1 expression levels, the mean CCAT1 expression level among all lung adenocarcinoma tissues (mean, 13.33) was used as the cutoff value. All 96 patients were divided into a low-CCAT1 expression group $(\mathrm{n}=60)$ and a high-CCAT1 expression group $(\mathrm{n}=56)$. CCAT1 expression was significantly associated with EGFR expression $(\mathrm{P}=0.011)$, lymphatic metastasis $(\mathrm{P}=0.003)$ and TNM stage $(\mathrm{P}=0.003)$, whereas no significant association with age or sex were observed (Table I). The expression of CCAT1 was then detected in lung adenocarcinoma cell lines (H358, H23, H1975, H1650, H1299 and A549) and a normal human bronchial epithelial cell line (16HBE). The RT-qPCR assay indicated that CCAT1 was higher in all lung adenocarcinoma cell lines than in the normal lung cell line ( $\mathrm{P}<0.01$; Fig. 1B). Based on CCAT1 expression patterns, the $\mathrm{H} 358$ and $\mathrm{H} 1650$ cell lines were selected to detect the effect of lncRNA CCAT1 in in vitro experiments.

CCAT1 regulates the migration and invasion of lung adenocarcinoma cell lines in vitro. To explore the biological functions of CCAT1 in the cell migration and invasion of lung adenocarcinoma, CCAT1 expression was upregulated in H358 cells by transfection with a pcDNA3.1-CCAT1 plasmid, and downregulated in H1650 cells by interfering with siRNA. The transfection efficiency was determined by RT-qPCR (Fig. 2A). A Transwell assay indicated that upregulation of CCAT1 promoted the migration of $\mathrm{H} 358$ cells, while suppression of CCAT1 inhibited the migration of H1650 cells, $(\mathrm{P}<0.01$; Fig. 2B). Furthermore, upregulated CCAT1 increased $\mathrm{H} 358$ cell invasion, while knockdown of CCAT1 suppressed H1650 cell invasion ( $\mathrm{P}<0.01$; Fig. $2 \mathrm{C}$ ). The results suggested that aberrant expression of CCAT1 acts to regulate lung adenocarcinoma cell metastasis.

IncRNA CCAT1 influences the expression of EMT-associated markers in lung adenocarcinoma. To explore whether aberrant CCAT1 expression levels were involved in the process of EMT, the mesenchymal markers $\mathrm{N}$-cadherin and vimentin, and the epithelial marker E-cadherin were investigated by western blotting. Overexpression of CCAT1 in H358 cells resulted in upregulation of $\mathrm{N}$-cadherin and vimentin protein expression and downregulation of E-cadherin protein; while knockdown of CCAT1 expression in H1650 cells resulted in downregulation 
A
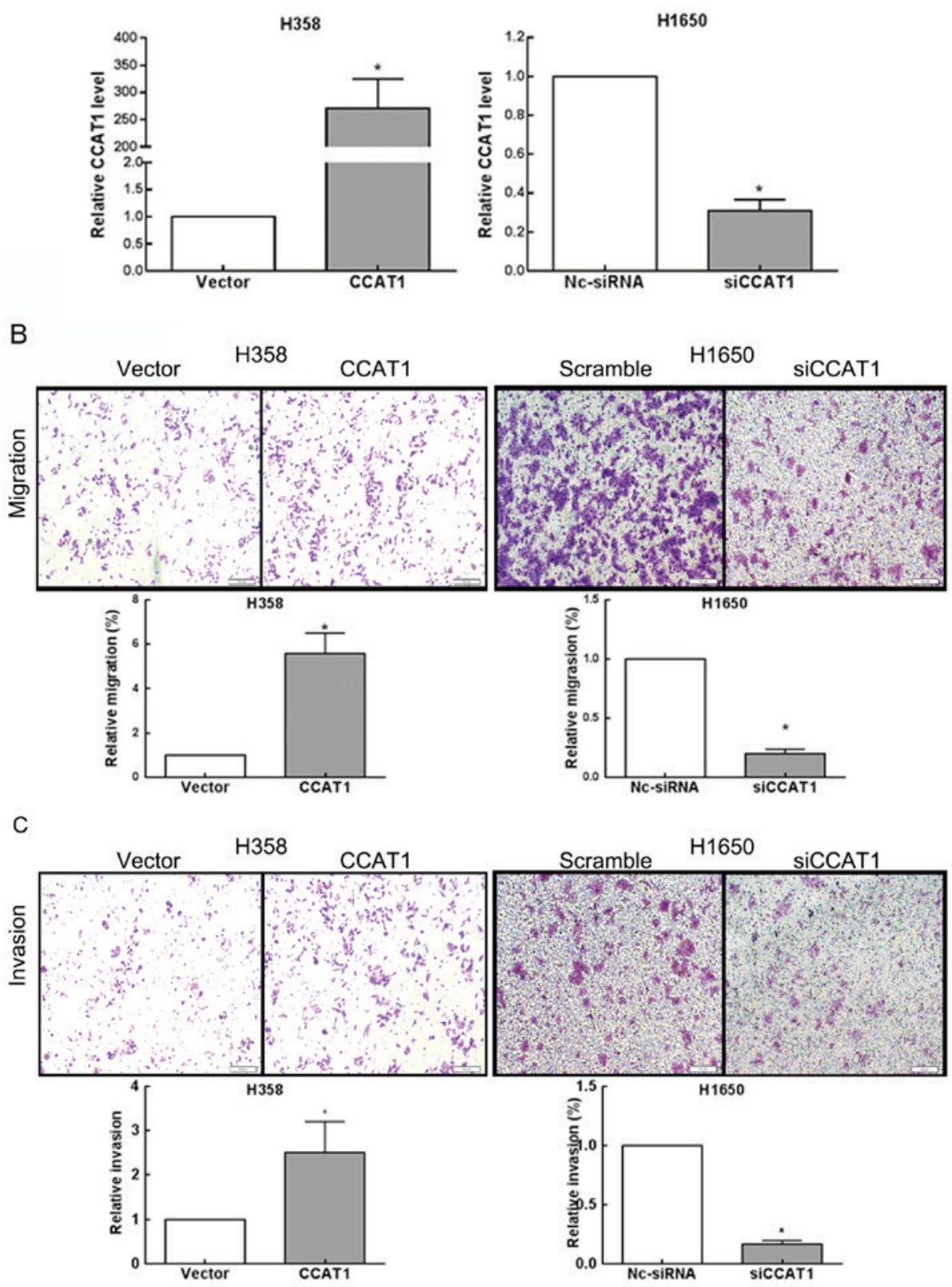

Figure 2. CCAT1 regulates cell migration and invasion in vitro. (A) CCAT1 expression levels were determined using reverse transcription-quantitative polymerase chain reaction in $\mathrm{H} 358$ and $\mathrm{H} 1650$ cells transfected with pcDNA3.1-CCAT1 or an empty vector. $\beta$-actin was used as an internal control. Corresponding P-values analyzed by Mann-Whitney U-test are indicated. (B) Representative images and quantification of Transwell migration assays for H358 and H1650 cells indicated that CCAT1 promotes cells migration in vitro (scale bar, $50 \mu \mathrm{m}$ ). "P<0.01. Corresponding P-values analyzed by Mann-Whitney U-test are indicated. (C) Representative images (up) and quantification (down) of Transwell invasion assays for H358 and H1650 cells demonstrated that CCAT1 promotes cells invasion in vitro (scale bar, $50 \mu \mathrm{m}$ ). ${ }^{*} \mathrm{P}<0.01$. Corresponding P-values analyzed by Mann-Whitney U-test are indicated. CCAT1, colon cancer-associated transcript 1; siCCAT1, small interfering RNA against colon cancer-associated transcript 1; Nc-siRNA, noncoding small interfering RNA.

of $\mathrm{N}$-cadherin and vimentin protein expression and upregulation of E-cadherin protein $(\mathrm{P}<0.01$; Fig. 3). This indicates that CCAT1 may contribute to the regulation of EMT-associated marker expression in lung adenocarcinoma cell lines.

\section{Discussion}

In the present study, the expression of the IncRNA CCAT1 and its biological effect on tumor cell metastasis in lung adenocarcinoma were detected and investigated, and it was confirmed that CCAT1 is upregulated in lung adenocarcinoma. Clinically, high expression levels of CCAT1 were associated with EGFR expression, lymphatic metastasis and tumor stage. The clinical results indicated that CCAT1 may be involved in the development of lung adenocarcinoma. Furthermore, upregulation of CCAT1 promoted the migration and invasion of lung adenocarcinoma cell lines, and downregulation of CCAT1 inhibited cell migration and invasion in vitro. Additionally, the results demonstrated that CCAT1 may induce EMT to promote cell metastasis in lung adenocarcinoma. 
A

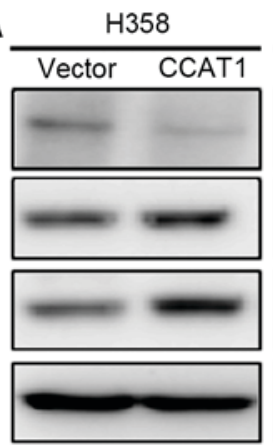

$\mathrm{H} 1650$

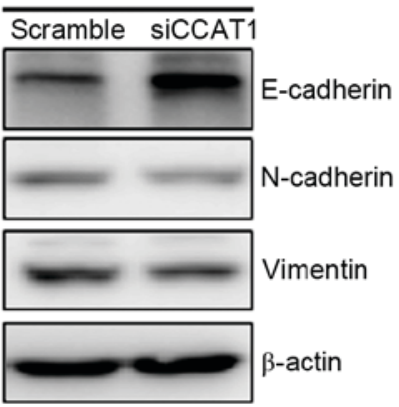

B

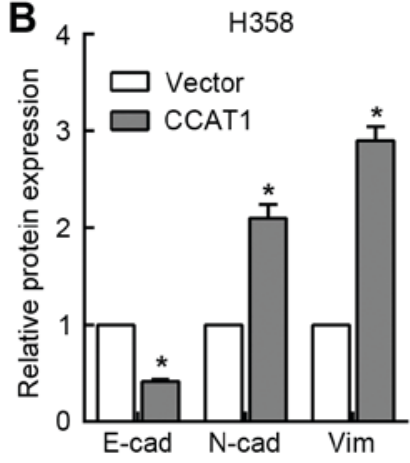

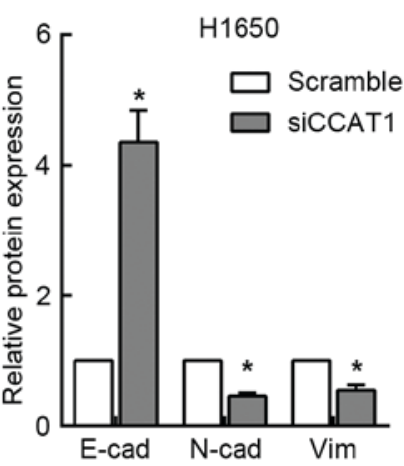

Figure 3. CCAT1 regulates the expression levels of epithelial-to-mesenchymal transition-associated markers. (A) N-cadherin, vimentin and E-cadherin protein expression levels in lung adenocarcinoma cells were analyzed by western blotting. CCAT1 overexpression promoted the expression of N-cadherin and vimentin, while suppressing E-cadherin expression in vitro. $\beta$-actin was used as a loading control. (B) Average values of integrated optical density of the bands were calculated by analyzing the results of five repeated experiments.. Data are presented as the mean \pm standard error of the mean. ${ }^{*}<0.01$. Corresponding P-values determined by Mann-Whitney U-test are indicated. CCAT1, colon cancer-associated transcript 1; siCCAT1, small interfering RNA against colon cancer-associated transcript 1; E-cad, E-cadherin, N-cad, N-cadherin; vim, vimentin.

IncRNAs have been reported to serve important regulatory roles in the biology of human malignancy (10). Specifically for lung adenocarcinoma, it has already been reported that lncRNAs contribute to the malignant phenotype of tumor cells (11-16). The lncRNA CCAT1 (NR_108049.1) was initially identified as a strongly upregulated noncoding transcript (2,795 bases in humans) in colon cancer, and high CCAT1 expression is associated with metastasis in colon cancer (17). Recently, Chen et al (18) identified that CCAT1 is responsible for docetaxel resistance in lung adenocarcinoma cells. The present study demonstrated that CCAT1 was significantly increased in the patient-derived lung adenocarcinoma tissues compared with the corresponding normal tissues, with the expression of CCAT1 in lung cancer cell lines further confirming the results. Additionally, due to the positive association between CCAT1 and high tumor burden (CCAT1 expression was significantly associated with lymphatic metastasis and TNM stage), cell migration and invasion assays were performed. The results suggested that upregulation of CCAT1 induced cell migration and invasion, while downregulation of CCAT1 inhibited cell metastasis, which meant that CCAT1 could promote the malignant phenotype of lung adenocarcinoma, with the results being consistent with those of Luo et al (19). Mechanistically, CCAT1 functions as an oncogene in lung adenocarcinoma through the upregulation of EMT-associated proteins.

EGFR status is a critical factor for the selection of therapeutic strategy in patients with lung adenocarcinoma. In the present study, the results indicated that CCAT1 expression was associated with EGFR status; as EGFR-mutated NSCLC patients can benefit from EGFR-tyrosine kinase inhibitor (TKI) therapies, genes associated with EGFR status may also act as pivotal therapeutic targets. The mechanisms underlying this relationship remain unclear; therefore future studies should focus on the role of CCAT1 in EGFR mutation, and whether it is involved in the development of resistance to EGFR-TKIs in lung cancer. Previous studies have implicated the activation of EMT in resistance to EGFR-TKIs; activation of EMT can suppress E-cadherin and directly reduce sensitivity to EGFR-TKIs $(20,21)$. As a secondary mutation, T790M is the most common mechanism for acquisition of EGFR-TKI resistance (in 50-60\% of cases) (22). Since CCAT1 is an EMT-promoting lncRNA, CCAT1 may also act as a promotion molecule overcoming EGFR-TKI resistance.

Previous studies have demonstrated that CCAT1 can interact with MYC enhancer in colorectal and prostate cancer and, in turn, regulate long-range chromatin interactions at the MYC locus (4), acting as an oncogene sponge in human gallbladder cancer and hepatocellular carcinoma, partly by repressing miRNAs $(8,23)$. CCAT1 can also promote the acquisition of chemoresistance and EMT phenotypes in docetaxel-resistant lung adenocarcinoma cells by competitively binding with let-7c (18). However, as the molecular mechanism of a particular lncRNA may differ in different tumor microenvironments, histological subtypes and tumor stages, CCAT1 may regulate lung adenocarcinoma cell metastasis and EMT phenotypes through different potential targets, and the specific target genes controlled by CCAT1 in lung adenocarcinoma require further studies for clarification.

Taken together, the findings of the present study indicate that aberrant expression of CCAT1 is associated with EGFR status, tumor metastasis and tumor stage in lung adenocarcinoma. In addition, overexpression of CCAT1 promotes metastasis via the induction of EMT in vitro. Further research may identify a way in which the pathway linking CCAT1 and EMT may be exploited as a therapeutic approach for the treatment of lung adenocarcinoma.

\section{Acknowledgements}

Not applicable.

\section{Funding}

No funding received.

\section{Availability of data and materials}

The datasets generated in the present study are available on reasonable request from the corresponding author. 


\section{Authors' contributions}

HL participated in the design of the study and performed the statistical analysis. XZ, WC and HY conceived the study, participated in its design and execution, and helped to draft the manuscript. All authors read and approved the final manuscript.

\section{Ethics approval and consent to participate}

Informed consent was obtained from each patient, and the study was approved by the Ethics Committee of The Third Affiliated Hospital of Wenzhou Medical University (Wenzhou, China).

\section{Consent for publication}

Consent for publication was obtained from the study participants.

\section{Competing interests}

The authors declare that they have no competing interests.

\section{References}

1. Chen W, Zheng R, Baade PD, Zhang S, Zeng H, Bray F, Jemal A, Yu XQ and He J: Cancer statistics in China, 2015. CA Cancer J Clin 66: 115-132, 2016.

2. Rosell R and Karachaliou N: Lung cancer: Maintenance therapy and precision medicine in NSCLC. Nat Rev Clin Oncol 10 549-550, 2013

3. Wood SL, Pernemalm M, Crosbie PA and Whetton AD: The role of the tumor-microenvironment in lung cancer-metastasis and its relationship to potential therapeutic targets. Cancer Treat Rev 40: 558-566, 2014.

4. Xiang JF, Yin QF, Chen T, Zhang Y, Zhang XO, Wu Z, Zhang S, Wang HB, Ge J, Lu X, et al: Human colorectal cancer-specific CCAT1-L lncRNA regulates long-range chromatin interactions at the MYC locus. Cell Res 24: 513-531, 2014.

5. Zhu HQ, Zhou X, Chang H, Li HG, Liu FF, Ma CQ and Lu J: Aberrant expression of CCAT1 regulated by c-Myc predicts the prognosis of hepatocellular carcinoma. Asian Pac J Cancer Prev 16: 5181-5185, 2015.

6. Wang F, Xie C, Zhao W, Deng Z, Yang H and Fang Q: Long non-coding RNA CARLo-5 expression is associated with disease progression and predicts outcome in hepatocellular carcinoma patients. Clin Exp Med 17: 33-43, 2017.

7. Yang F, Xue X, Bi J, Zheng L, Zhi K, Gu Y and Fang G: Long noncoding RNA CCAT1, which could be activated by c-Myc, promotes the progression of gastric carcinoma. J Cancer Res Clin Oncol 139: 437-445, 2013.

8. Kim T, Cui R, Jeon YJ, Lee JH, Lee JH, Sim H, Park JK, Fadda P, Tili E, Nakanishi H, et al: Long-range interaction and correlation between MYC enhancer and oncogenic long noncoding RNA CARLo-5. Proc Natl Acad Sci USA 111: 4173-4178, 2014.
9. Livak KJ and Schmittgen TD: Analysis of relative gene expression data using real-time quantitative PCR and the 2(-Delta Delta C(T)) method. Methods 25: 402-408, 2001.

10. Beermann J, Piccoli MT, Viereck J and Thum T: Non-coding RNAs in development and disease: Background, mechanisms, and therapeutic approaches. Physiol Rev 96: 1297-1325, 2016.

11. Yang YR, Zang SZ, Zhong CL, Li YX, Zhao SS and Feng XJ: Increased expression of the lncRNA PVT1 promotes tumorigenesis in non-small cell lung cancer. Int J Clin Exp Pathol 7: 6929-6935, 2014.

12. Nie FQ, Sun M, Yang JS, Xie M, Xu TP, Xia R, Liu YW, Liu XH, Zhang EB, Lu KH and Shu YQ: Long noncoding RNA ANRIL promotes non-small cell lung cancer cell proliferation and inhibits apoptosis by silencing KLF2 and P21 expression. Mol Cancer Ther 14: 268-277, 2015.

13. Nie W, Ge HJ, Yang XQ, Sun X, Huang H, Tao X, Chen WS and Li B: LncRNA-UCA1 exerts oncogenic functions in non-small cell lung cancer by targeting miR-193a-3p. Cancer Lett 371: 99-106, 2016.

14. Shen L, Chen L, Wang Y, Jiang X, Xia H and Zhuang Z: Long noncoding RNA MALAT1 promotes brain metastasis by inducing epithelial-mesenchymal transition in lung cancer. J Neurooncol 121: 101-108, 2015.

15. Wang G, Chen H and Liu J: The long noncoding RNA LINC01207 promotes proliferation of lung adenocarcinoma. Am J Cancer Res 5: 3162-3173, 2015.

16. Wu Y, Liu H, Shi X, Yao Y, Yang W and Song Y: The long non-coding RNA HNF1A-AS1 regulates proliferation and metastasis in lung adenocarcinoma. Oncotarget 6: 9160-9172, 2015.

17. Alaiyan B, Ilyayev N, Stojadinovic A, Izadjoo M, Roistacher M, Pavlov V, Tzivin V, Halle D, Pan H, Trink B, et al: Differential expression of colon cancer associated transcript1 (CCAT1) along the colonic adenoma-carcinoma sequence. BMC Cancer 13: 196, 2013.

18. Chen J, Zhang K, Song H, Wang R, Chu X and Chen L: Long noncoding RNA CCAT1 acts as an oncogene and promotes chemoresistance in docetaxel-resistant lung adenocarcinoma cells. Oncotarget 7: 62474-62489, 2016.

19. Luo J, Tang L, Zhang J, Ni J, Zhang HP, Zhang L, Xu JF and Zheng D: Long non-coding RNA CARLo-5 is a negative prognostic factor and exhibits tumor pro-oncogenic activity in non-small cell lung cancer. Tumour Biol 35: 11541-11549, 2014

20. Hampton KK and Craven RJ: Pathways driving the endocytosis of mutant and wild-type EGFR in cancer. Oncoscience 1: 504-512, 2014.

21. Rolfo C, Giovannetti E, Hong DS, Bivona T, Raez LE, Bronte G, Buffoni L, Reguart N, Santos ES, Germonpre P, et al: Novel therapeutic strategies for patients with NSCLC that do not respond to treatment with EGFR inhibitors. Cancer Treat Rev 40: 990-1004, 2014.

22. Sequist LV, Waltman BA, Dias-Santagata D, Digumarthy S, Turke AB, Fidias P, Bergethon K, Shaw AT, Gettinger S, Cosper AK, et al: Genotypic and histological evolution of lung cancers acquiring resistance to EGFR inhibitors. Sci Trans1 Med 3: 75ra26, 2011.

23. Ma MZ, Chu BF, Zhang Y, Weng MZ, Qin YY, Gong W and Quan ZW: Long non-coding RNA CCAT1 promotes gallbladder cancer development via negative modulation of miRNA-218-5p. Cell Death Dis 6: e1583, 2015. 